Article

\title{
Optimal Control of a Multilayer Electroelastic Engine with a Longitudinal Piezoeffect for Nanomechatronics Systems
}

\author{
Sergey M. Afonin \\ National Research University of Electronic Technology (MIET), 124498 Moscow, Russia; learner01@mail.ru
}

Received: 21 May 2020; Accepted: 25 November 2020; Published: 1 December 2020

\begin{abstract}
A electroelastic engine with a longitudinal piezoeffect is widely used in nanotechnology for nanomanipulators, laser systems, nanopumps, and scanning microscopy. For these nanomechatronics systems, the transition between individual positions of the systems in the shortest possible time is relevant. It is relevant to solve the problem of optimizing the nanopositioning control system with a minimum control time. This work determines the optimal control of a multilayer electroelastic engine with a longitudinal piezoeffect and minimal control time for an optimal nanomechatronics system. The expressions of the control function and switching line are obtained with using the Pontryagin maximum principle for the optimal control system of the multilayer electroelastic engine at a longitudinal piezoeffect with an ordinary second-order differential equation of system. In this optimal nanomechatronics system, the control function takes only two values and changes once.
\end{abstract}

Keywords: multilayer electroelastic engine; longitudinal piezoeffect; optimal control; Pontryagin maximum principle; nanomechatronics system; ordinary second-order differential equation; control function; switching line

\section{Introduction}

An electroelastic actuator in the form piezo actuator is used for nanotechnology in nanomanipulators, laser systems, nanopumps, scanning microscopy [1]. The piezo actuator is used in photolithography for nano- and microdisplacements when aligning templates, in medical equipment for precise instrument delivery during microsurgical operations, in optical-mechanical devices, in adaptive optics systems, and in adaptive telescopes [2]. It is also used in stabilization systems for optical-mechanical devices, systems for alignment and tuning of lasers, interferometers, adaptive optical systems and fiber-optic systems for transmitting and receiving information [3].

The application of a multilayer electroelastic engine with a longitudinal piezoeffect is promising for nanomanipulators in nanotechnology [4]. To increase the range of the displacement, a cellular actuator and a multilayer electroelastic engine are used [5]. Mechatronics control systems with a multilayer electroelastic engine with a longitudinal piezoeffect are used in precision engineering [6].

It is important to solve the problem of positioning a multilayer electroelastic engine with a longitudinal piezoeffect from an arbitrary state with minimal control time [7] with the transition between individual positions of the optimal control system in the shortest possible time. The Pontryagin maximum principle is used to derive minimum time control. The prospects for constructing optimal control systems for nanomechatronics are shown in work [8].

In this work using the Pontryagin maximum principle for optimal systems, which ensures under optimal control the maximum of the Hamilton function and the minimum of the control time. The expression of the control function is obtained, which has only two values and changes once. 
The switching line is obtained for the optimal control of a multilayer electroelastic engine with a longitudinal piezoeffect in a nanomechatronics optimal system.

\section{Optimal Control of a Multilayer Electroelastic Engine}

\subsection{Characteristics of a Multilayer Electroelastic Engine with a Longitudinal Piezoeffect}

Let us consider a multilayer electroelastic engine with a longitudinal piezoeffect with one fixed face in Figure 1, where $P$ is the polarization and 3 is the axis and $S_{3}(t)$ is the relative deformation along axis 3 and $t$ is time. The equation for the inverse longitudinal piezoeffect [9] has the form

$$
S_{3}(t)=d_{33} E_{3}(t)+s_{33}^{E} T_{3}(t)
$$

where $d_{33}$ is the longitudinal piezomodule, $E_{3}(t)$ is the electric field tension along axis $3, s_{33}^{E}$ is the elastic compliance at $E=$ const, and $T_{3}(t)$ is the mechanic tension along axis 3 . From the inverse longitudinal piezoeffect Equation (1), the adjusting characteristic of a multilayer electroelastic engine is found for elastic load in the form

$$
\begin{gathered}
\frac{\xi(t)}{l}=d_{33} E_{3}(t)-\frac{s_{33}^{E} C_{e} \xi(t)}{S_{0}} \\
l=n \delta, F(t)=C_{e} \xi(t), E_{3}(t)=U(t) / \delta
\end{gathered}
$$

where $\xi(t)$ is the displacement of the face engine, $t$ is time, $l$ is the length of the engine, $n$ is the number of piezolayers, $\delta$ is the thickness of the piezolayer, $C_{e}$ the stiffness of the load, $S_{0}$ is the area of the engine, $F(t)$ is the force, and $U(t)$ is the voltage.

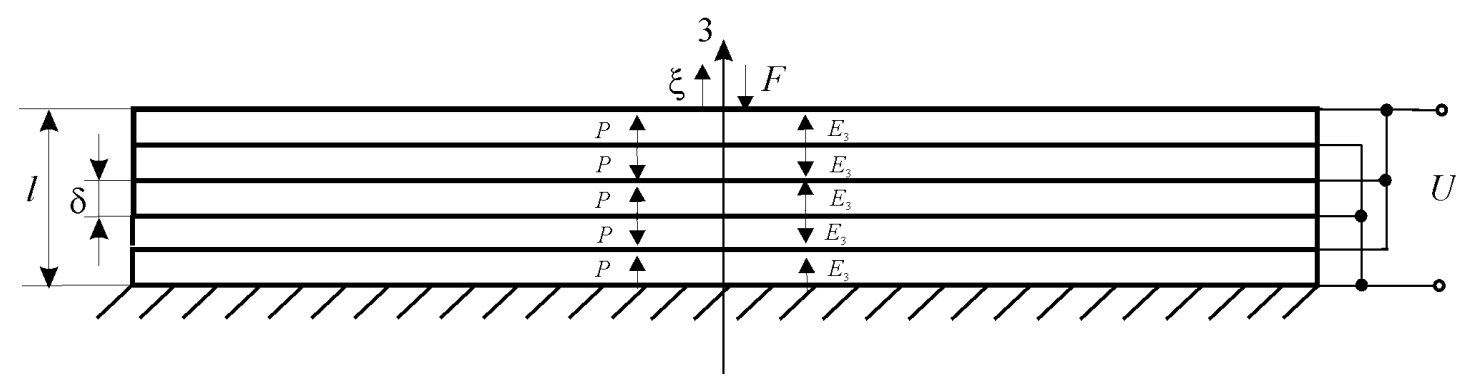

Figure 1. The kinematic scheme of a multilayer electroelastic engine with a longitudinal piezoeffect.

The decisions the characteristics for the piezo engine are obtained in the works [10-17]. Therefore, for the multilayer electroelastic engine at longitudinal piezoeffect the adjusting characteristic has the following form:

$$
\begin{gathered}
\xi(t)=\frac{d_{33} n U(t)}{1+C_{e} / C_{33}^{E}}=b_{33}^{U} U(t) \\
C_{33}^{E}=S_{0} /\left(s_{33}^{E} l\right), \xi(t) / U(t)=b_{33}^{U}=d_{33} n /\left(1+C_{e} / C_{33}^{E}\right)
\end{gathered}
$$

where $C_{33}^{E}$ and $b_{33}^{U}$ are the stiffness of the multilayer electroelastic engine with a longitudinal piezoeffect and the transfer coefficient, respectively. The multilayer piezo engine with a longitudinal piezoeffect from a ceramic PZT at $d_{33}=4 \times 10^{-10} \mathrm{~m} / \mathrm{V}, n=5, C_{33}^{E}=12 \times 10^{7} \mathrm{~N} / \mathrm{m}, C_{e}=0.6 \times 10^{7} \mathrm{~N} / \mathrm{m}, U=100 \mathrm{~V}$ results in $b_{33}^{U}=1.9 \mathrm{~nm} / \mathrm{V}$ and $\xi=190 \mathrm{~nm}$.

In a control system the fransfer function $W_{n}(s)$ multilayer electroelastic engine with a longitudinal piezoeffect is determined in the form

$$
W_{n}(s)=\frac{\Xi(s)}{U(s)}=\frac{b_{33}^{U}}{T_{n} s+1}
$$




$$
T_{n}=R C_{n}
$$

where $\Xi(s)$ and $U(s)$ are Laplace displacement $\xi(t)$ and voltage $U(t)$ in Figure $1, s$ is the transformation operator, $T_{n}$ is the electrical constant time of the multilayer electroelastic engine, $C_{n}$ is the capacitance of the multilayer electroelastic engine, and $R$ is the matching circuit resistance. For the multilayer piezo engine with a longitudinal piezoeffect from a ceramic PZT at $R=10 \mathrm{kOm}$ and $C_{n}=0.5 \mu \mathrm{F}$, the electrical constant time is $T_{n}=5 \mathrm{~ms}$.

Let us consider the multilayer electroelastic engine with a longitudinal piezoeffect and the electrical constant time is much larger than the mechanical constant time [10] of the multilayer engine at an elastic inertial load:

$$
\begin{aligned}
T_{m}= & \sqrt{M /\left(C_{e}+C_{33}^{E}\right)} \\
& T_{n} \gg>T_{m}
\end{aligned}
$$

where $T_{m}$ and $M$ are the mechanical constant time of the engine at an elastic inertial load and the mass of the load, respectively. For the multilayer piezo engine with a longitudinal piezoeffect from a ceramic PZT at an elastic inertial load $M=0.3 \mathrm{~kg}, C_{33}^{E}=12 \times 10^{7} \mathrm{~N} / \mathrm{m}$, and $C_{e}=0.6 \times 10^{7} \mathrm{~N} / \mathrm{m}$, the mechanical constant time is $T_{m}=0.05 \mathrm{~ms}$.

Let us consider the optimal control system with the integrator in a series with the multilayer electroelastic engine with a longitudinal piezoeffect. In this optimal control system, the integrator transfer function $W_{i}(s)$ has the form

$$
\begin{aligned}
W_{i}(s) & =\frac{k_{i}}{s} \\
T_{n}>>T_{i} & =1 / k_{i}
\end{aligned}
$$

where $k_{i}$ is the transfer coefficient of the integrator.

Accordingly, for the control system with the integrator for the multilayer electroelastic engine with a longitudinal piezoeffect, considering the electrical constant time of the multilayer electroelastic engine and the transfer coefficient of the integrator, the ordinary second-order differential equation is written as

$$
\begin{gathered}
T_{n} \frac{d^{2} \xi(t)}{d t^{2}}+\frac{d \xi(t)}{d t}=k_{33}^{U} u(t) \\
k_{33}^{U}=k_{i} b_{33^{\prime}}^{U},|u| \leq u_{0}
\end{gathered}
$$

where $\xi(t), u(t)$, and $k_{33}^{U}$ are the displacement, the voltage, and transfer coefficient of the control system of the multilayer electroelastic engine with a longitudinal piezoeffect, respectively.

For the ordinary second-order differential Equation (6), therefore, the system of differential equations in Cauchy form is obtained,

$$
\left\{\begin{array}{l}
\frac{d \xi_{1}}{d t}=\xi_{2} \\
\frac{d \xi_{2}}{d t}=-\frac{1}{T_{n}} \xi_{2}+\frac{k_{33}^{U}}{T_{n}} u
\end{array}\right.
$$

where $\xi_{1}=\xi$ and $\xi_{2}=\dot{\xi}_{1}$ as the state variables are selected the displacement and the velocity of the multilayer electroelastic engine with a longitudinal piezoeffect.

2.2. Application of Pontryagin Maximum Principle for Decision Optimal Control of a Multilayer Electroelastic Engine

Using the Pontryagin maximum principle [18], the control law ensures the transition of the multilayer electroelastic engine from any initial state to a given end point at a minimum time. 
The end point is the origin of coordinates. The Hamilton function for this maximum principle has the following form

$$
\max _{u} H=\max _{u}\left(\psi_{1} \xi_{2}+\psi_{2}\left(-\frac{1}{T_{n}} \xi_{2}+\frac{k_{33}^{U}}{T_{n}} u\right)\right)
$$

where $\psi_{1}$ and $\psi_{2}$ are the functions.

Therefore, for the Hamilton function [19], the system of equations has the form

$$
\left\{\begin{array}{l}
\frac{d \psi_{1}}{d t}=-\frac{\partial H}{\partial \xi_{1}}=0 \\
\frac{d \psi_{2}}{d t}=-\frac{\partial H}{\partial \xi_{2}}=-\psi_{1}+\frac{1}{T_{n}} \psi_{2}
\end{array}\right.
$$

The solution to this system of equations has the form

$$
\left\{\begin{array}{l}
\psi_{1}=A_{1}=\text { const } \\
\psi_{2}=-A_{1} t+A_{2} e^{\frac{t}{T_{n}}}
\end{array}\right.
$$

Therefore, the function $\psi_{2}$ changes once the sign (see Figure 2), since the straight line and the exponent intersect once. According to the maximum principle, from (9) the control signal has two values, which equal in absolute value and opposite in the sign. The sign of the control signal coincides with the sign of this function $\psi_{2}$ on Figure 2. The control function $u$ has the form

$$
u=u_{0} \operatorname{sign} \psi_{2}
$$

where $u_{0}$ is the control signal amplitude.

In this optimal system with an ordinary second-order differential equation, in Figure $2, u$ takes only two values, $u_{0}$ and $-u_{0}$, and changes once.

The trajectory of the control system of the multilayer electroelastic engine with a longitudinal piezoeffect in the phase plane consists of two sections, the initial and the final, with the latter passing through the origin and acts as the switching line. The switching line of the multilayer electroelastic engine is defined on the phase plane.

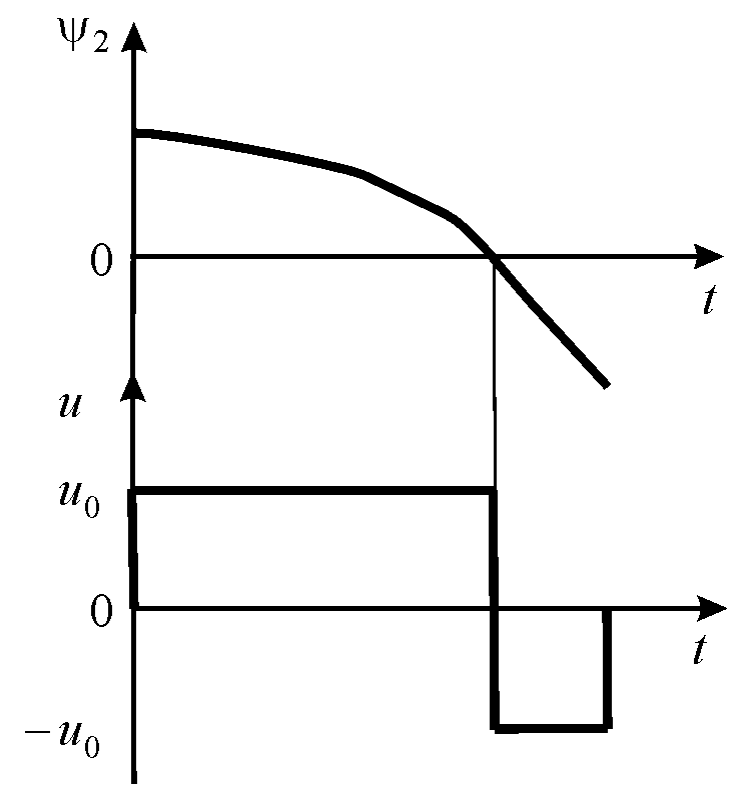

Figure 2. The control function in the optimal system. 
The solution for the system of Equation (8) for $u=$ const has the form

$$
\left\{\begin{array}{l}
\xi_{1}=k_{33}^{U} u t-T_{n} B_{2} e^{-\frac{t}{T_{n}}}+B_{1} \\
\xi_{2}=k_{33}^{U} u+B_{2} e^{-\frac{t}{T_{n}}}
\end{array},\right.
$$

when the initial location of the system is at the origin and when the time is $\tau=-t$, the constants are found in the form

$$
\left\{\begin{array}{l}
B_{1}=-k_{33}^{U} u T_{n} \\
B_{2}=-k_{33}^{U} u
\end{array},\right.
$$

then, the system of equations is determined in the form

$$
\left\{\begin{array}{l}
\xi_{1}=-k_{33}^{U} u \tau+k_{33}^{U} u T_{n} e^{\frac{\tau}{T_{n}}}-k_{33}^{U} u T_{n} \\
\xi_{2}=k_{33}^{U} u\left(1-e^{\frac{\tau}{T_{n}}}\right) .
\end{array} .\right.
$$

From (15), the equation has the form

$$
e^{\frac{\tau}{T_{n}}}=1-\frac{\xi_{2}}{k_{33}^{U} u} .
$$

Accordingly, the time is found in the form

$$
\tau=T_{n} \ln \left(1-\frac{\xi_{2}}{k_{33}^{U} u}\right) .
$$

At the parameters $\xi_{1}=0$ and $\xi_{2}=0$, the time is obtained $\tau=0$.

Therefore, the equation for the final portion of the movement trajectory of the multilayer electroelastic engine with a longitudinal piezoeffect is obtained on the phase plane in the form of the switching line:

$$
\xi_{1}=-k_{33}^{U} u T_{n} \ln \left(1-\frac{\xi_{2}}{k_{33}^{E} u}\right)+k_{33}^{U} u T_{n}\left(1-\frac{\xi_{2}}{k_{33}^{E} u}\right)-k_{33}^{U} u T_{n} .
$$

From (8), the control function is found in the form

$$
u=-u_{0} \operatorname{sign} \xi_{2} .
$$

The switching line or the last section of the trajectory divides the phase plane into two regions at $s>0$ and $s<0$, When each area of management is constant, a change in the control occurs at $s=0$. Accordingly, we choose a control for each region so that the point on the phase plane moves toward the switching line.

Then we obtain the expression (19) for the equation into Equation (18) for the last section of the movement trajectory of the multilayer electroelastic engine with a longitudinal piezoeffect. Accordingly, the equation for the last section of the movement trajectory is obtained in the following form:

$$
s\left(\xi_{1}, \xi_{2}\right)=\xi_{1}-k_{33}^{U} u_{0} T_{n} \operatorname{sign} \xi_{2} \ln \left(1+\frac{\left|\xi_{2}\right|}{k_{33}^{U} u_{0}}\right)+T_{n} \xi_{2}=0 .
$$

Therefore, the equation for the last section of the trajectory has the form

$$
\xi_{1}=k_{33}^{U} u_{0} T_{n} \operatorname{sign} \xi_{2} \ln \left(1+\frac{\left|\xi_{2}\right|}{k_{33}^{U} u_{0}}\right)-T_{n} \xi_{2} .
$$


Then the inequality is obtained in the form

$$
\frac{d\left|s\left(\xi_{1}, \xi_{2}\right)\right|}{d t} \leq 0 .
$$

Therefore, the inequality is written as

$$
\frac{d s\left(\xi_{1}, \xi_{2}\right)}{d t} \operatorname{signs}\left(\xi_{1}, \xi_{2}\right) \leq 0 .
$$

Substituting expression (21) for the function $s\left(\xi_{1}, \xi_{2}\right)$ into expression (23) and considering system (8), the condition has the form

$$
\frac{\xi_{2} k_{33}^{U} u_{0}}{k_{33}^{U} u_{0}+\left|\xi_{2}\right|} \operatorname{signs}+\frac{\left|\xi_{2}\right| k_{33}^{U}}{k_{33}^{U} u_{0}+\left|\xi_{2}\right|} u \text { signs } \leq 0
$$

The second term of expression (24) depends on the control. Accordingly, condition (24) is satisfied under the control of the form

$$
u=-u_{0} \text { signs }
$$

Therefore, in this system with an ordinary second-order differential equation, the optimal control takes only two values and changes once. The expressions of the control function and switching line are obtained with using the Pontryagin maximum principle for the optimal control system of the multilayer electroelastic engine at a longitudinal piezoeffect with an ordinary second-order differential equation of system.

\section{Conclusions}

The optimal control of the multilayer electroelastic engine with a longitudinal piezoeffect and a minimal control time is obtained for nanomechatronics systems. The expressions of the control function and switching line on the phase plane are determined for the optimal control of this multilayer electroelastic engine. Using the Pontryagin maximum principle, the control law is obtained for the transition of the multilayer electroelastic engine from any initial state to a given end point in a minimal amount of time. The optimal control of the multilayer electroelastic engine with an ordinary second-order differential equation takes only two values and changes once.

Funding: This research received no external funding.

Conflicts of Interest: The authors declare no conflict of interest.

\section{References}

1. Uchino, K. Piezoelectric Actuator and Ultrasonic Motors; Kluwer Academic Publisher: Boston, MA, USA, 1997; 347p.

2. Afonin, S.M. Absolute stability conditions for a system controlling the deformation of an elecromagnetoelastic transduser. Dokl. Math. 2006, 74, 943-948. [CrossRef]

3. Afonin, S.M. Structural-parametric model and transfer functions of electroelastic actuator for nanoand microdisplacement. In Piezoelectrics and Nanomaterials: Fundamentals, Developments and Applications; Parinov, I.A., Ed.; Nova Science: New York, NY, USA, 2015; Chapter 9, pp. 225-242.

4. Afonin, S.M. A structural-parametric model of electroelastic actuator for nano- and microdisplacement of mechatronic system. In Advances in Nanotechnology; Bartul, Z., Trenor, J., Eds.; Nova Science: New York, NY, USA, 2017; Chapter 8, Volume 19, pp. 259-284.

5. Schultz, J.; Ueda, J.; Asada, H. Cellular Actuators; Butterworth-Heinemann Publisher: Oxford, UK, 2017; 382p.

6. Karpelson, M.; Wei, G.-Y.; Wood, R.J. Driving high voltage piezoelectric actuators in microrobotic applications. Sens. Actuators A 2012, 176, 78-89. [CrossRef]

7. Belfiore, N.P. Micromanipulation: A Challenge for Actuation. Actuators 2018, 7, 85. [CrossRef] 
8. Bonciani, G.; Biancucci, G.; Fioravanti, S.; Valiyev, V.; Binni, A. Learning Micromanipulation, Part 2: Term Projects in Practice. Actuators 2018, 7, 56. [CrossRef]

9. Berlincourt, D.A.; Curran, D.R.; Jaffe, H. Piezoelectric and piezomagnetic materials and their function in transducers. In Physical Acoustics: Methods and Devices. Volume 1. Part A; Mason, W.P., Ed.; Academic Press: New York, NY, USA, 1964; Chapter 3, pp. 169-270.

10. Afonin, S.M. Structural-parametric model and diagram of a multilayer electromagnetoelastic actuator for nanomechanics. Actuators 2019, 8, 52. [CrossRef]

11. Afonin, S.M. Structural-parametric model electromagnetoelastic actuator nanodisplacement for mechatronics. Int. J. Phys. 2017, 5, 9-15. [CrossRef]

12. Afonin, S.M. Block diagrams of a multilayer piezoelectric motor for nano- and microdisplacements based on the transverse piezoeffect. J. Comput. Syst. Sci. Int. 2015, 54, 424-439. [CrossRef]

13. Afonin, S.M. Structural-parametric model of electromagnetoelastic actuator for nanomechanics. Actuators 2018, 7, 6. [CrossRef]

14. Afonin, S.M. Structural parametric model of a piezoelectric nanodisplacement transduser. Dokl. Phys. 2008, 53, 137-143. [CrossRef]

15. Afonin, S.M. A block diagram of electromagnetoelastic actuator nanodisplacement for communications Systems. Trans. Netw. Commun. 2018, 6, 1. [CrossRef]

16. Afonin, S.M. Structural-parametric model multilayer electromagnetoelastic actuator for nanomechatronics. Int. J. Phys. 2019, 7, 50-57. [CrossRef]

17. Afonin, S.M. Decision matrix equation and block diagram of multilayer electromagnetoelastic actuator micro and nanodisplacement for communications systems. Trans. Netw. Commun. 2019, 7, 11-21. [CrossRef]

18. Pontryagin, L.S.; Boltyanskii, V.G.; Gamkrelidze, R.V.; Mishchenko, E.F. The Mathematical Theory of Optimal Processes; Interscience Publishers John Wiley \& Sons, Inc.: New York, NY, USA; London, UK, 1962; 360p.

19. Gamkrelidze, R.V. Discovery of the maximum principle. J. Dyn. Control Syst. 1999, 5, 437-451. [CrossRef]

Publisher's Note: MDPI stays neutral with regard to jurisdictional claims in published maps and institutional affiliations.

(C) 2020 by the author. Licensee MDPI, Basel, Switzerland. This article is an open access article distributed under the terms and conditions of the Creative Commons Attribution (CC BY) license (http://creativecommons.org/licenses/by/4.0/). 